\title{
Low-energy electron collisions with ethane
}

\author{
M.H.F. Bettega* \\ Departamento de Física, Universidade Federal do Paraná, \\ Caixa Postal 19044, 81531-990, Curitiba, Paraná, Brazil \\ R.F. da Costa $^{\dagger}$ \\ Centro de Ciências Naturais e Humanas, Universidade Federal do ABC, \\ Rua Santa Adélia, 166, 09210-170 Santo André, São Paulo, Brazil \\ M.A.P. Lima \\ Centro de Ciência e Tecnologia do Bioetanol CTBE, Caixa Postal 6170, \\ 13083-970, Campinas, São Paulo, Brazil and Instituto de Física Gleb Wataghin, \\ Universidade Estadual de Campinas, Caixa Postal 6165, 13083-970, Campinas, SP, Brazil
}

(Received on 20 November, 2008)

\begin{abstract}
We employed the Schwinger multichannel method to compute elastic cross sections for low-energy electron collisions with ethane $\left(\mathrm{C}_{2} \mathrm{H}_{6}\right)$. The calculations were carried out in the static-exchange and static-exchange plus polarization approximations for energies up to $12 \mathrm{eV}$. Our integral cross section shows good agreement with experimental data and with theoretical results for energies above $5 \mathrm{eV}$. There are some differences for energies below $5 \mathrm{eV}$ between our results and the available experimental and theoretical results. Our differential cross sections also agree well with the experiment and with theory for energies above $5 \mathrm{eV}$; below this energy our results agree in shape, but are smaller than the available experimental and theoretical results. We discuss possible reasons for these discrepancies. We found a broad structure in the integral cross section around $8.5 \mathrm{eV}$ and also a Ramsauer-Townsend minimum around $0.2 \mathrm{eV}$. These results are in agreement with the experimental observations and theoretical results.
\end{abstract}

Keywords: Elastic scattering; Polarization effects; Ramsauer-Townsend minimum

\section{INTRODUCTION}

The recent discovery by Boudaïfa et al. [1] that secondary electrons are responsible for single and double strand breaks in DNA has motivated several studies regarding electron collisions with biological molecules [2]. Most of these studies are concerning with formation of resonances that would be responsible for molecular dissociation. Therefore the correct characterization of a resonance, i.e., its position, symmetry, type (shape, core-excited, Feshbach etc) is an information that helps in the understanding of the dissociation process.

Ethane is a simple hydrocarbon that has been studied by experimental [3-14] and theoretical groups [15-17]. This molecule presents a broad structure around $7 \mathrm{eV}$ and a Ramsauer-Townsend minimum below $1 \mathrm{eV}$ (at $\sim 0.2 \mathrm{eV}$ ) $[13,14,16]$. From the theoretical point of view the description of these two features needs the inclusion of polarization effects. These effects arise from the distortion of the molecular electronic cloud due to the electric field generated by the incoming electron. In order to obtain good cross sections for electron collisions with biological molecules, one needs to know how to incorporate polarization effects properly. Therefore, studies involving collisions with small molecules can help one to achieve this goal.

In this paper we report cross sections for elastic collisions of low-energy electrons with $\mathrm{C}_{2} \mathrm{H}_{6}$ molecules. We em-

\footnotetext{
*Electronic address: bettega@fisica.ufpr.br

†Electronic address: roma@ifi.unicamp.br

†lectronic address: maplima@ifi.unicamp.br
}

ployed the Schwinger multichannel (SMC) method implemented with norm-conserving pseudopotentials in the fixed nuclei, static-exchange (SE) and static-exchange plus polarization (SEP) approximations. We calculated integral, momentum transfer and differential cross sections for energies up to $12 \mathrm{eV}$. Our aim is to present results obtained with the inclusion of polarization effects that display the RamsauerTownsend minimum at the right location and to describe the correct shape of the differential cross sections. We compared our results with experimental and theoretical results available in the literature. In general our results agree well with the experimental data and theoretical results for energies above 5 $\mathrm{eV}$.

In the next sections we discuss the theoretical procedures used in the current calculations, present our results and discussions and end with a brief summary of our findings.

\section{THEORY}

The SMC method and its implementation with pseudopotentials (SMCPP) were discussed in detail elsewhere [18, 19]. Here we will only discuss the relevant aspects of the present calculation.

The target was computed in the Hartree-Fock approximation. Bound state and scattering calculations were done within the $C_{s}$ point group at the experimental geometry of equilibrium [20]. The geometrical structure of ethane is shown in Figure 1, generated by McMolPlt [21]. Our calculations employed the norm-conserving pseudopotentials of Bachelet, Hamman and Schlüter [22] in order to represent the core electrons, as discussed in Ref. [19].

The basis set employed in our bound state and scattering 


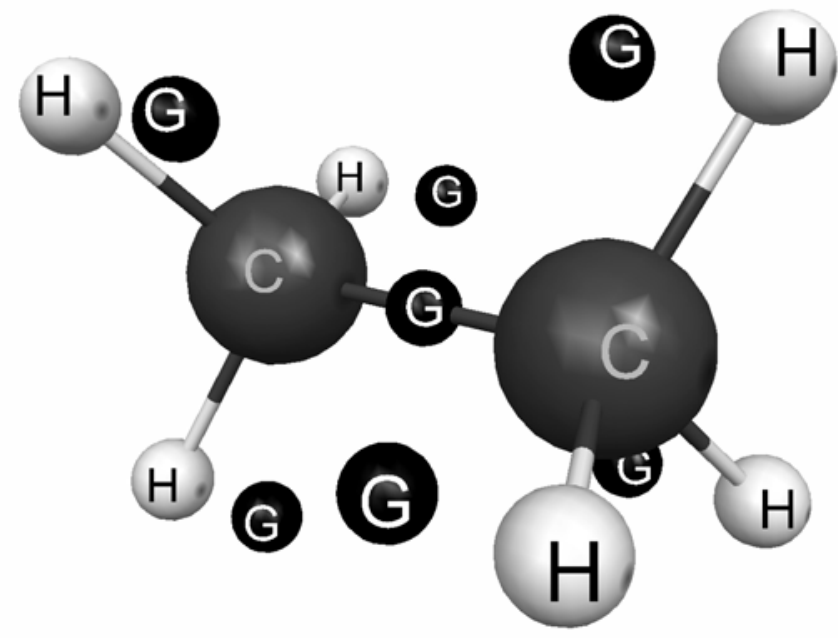

FIG. 1: Geometrical structure of ethane. The dark centers labeled as $G$ are extra (chargeless) centers used to locate additional Cartesian Gaussian functions in order to improve the one-particle basis set. See text for discussion.

calculations consists of Cartesian Gaussian functions generated by a variational method [23]. For carbon atoms the basis set consists of $6 s 5 p 2 d$ uncontracted Cartesian Gaussian functions with exponents: 12.49408, 2.470291, 0.614027, $0.184029,0.036799,0.013682$ for the $s$-type functions; $5.228869,1.592058,0.568612,0.210326,0.072250$, for the $p$-type functions and $0.603592,0.156753$ for the $d$-type functions. For the hydrogen atoms we used the $3 s$ basis set of Dunning [24], augmented by one $p$-type function with exponent 0.75 .

Recent calculations on electron-molecule collisions indicate that the most efficient way to take polarization effects into account is through the use of the modified virtual orbitals (MVOs) [25]. In the present calculations we used the modified virtual orbitals (MVOs) [26] to describe hole, particle and scattering orbitals according to Ref. [25]. We considered single excitations from all valence occupied orbitals to the MVOs with energies less than 15 hartree; the same set of MVOs were then used as scattering orbitals. We considered singlet and triplet coupled excitations which resulted in a total of 19,940 doublet configuration state functions (CSFs) divided per symmetry as follows: 10,460 for $A^{\prime}$ and 9,480 for $A^{\prime \prime}$. The one particle basis set used in these calculations included 114 primitive Cartesian Gaussian functions contracted to 102 functions. Recent calculations on electronpropane collisions have shown that it is important to include both singlet- and triplet-coupled excitations in order to obtain good results for low energies [27].

In order to improve the one-particle basis set, we performed another round of calculations including additional Cartesian Gaussian functions on seven extra (chargeless) centers. One center was located in the molecular center of mass, with one $s$-type function with exponent 0.5 and one $p$-type function with exponent 0.85 , and six remaining centers were placed out of the molecular axis. These six centers were located in the corners of two triangles parallel to the hydrogen's triangles. These two extra triangles were located in the half way between the center of mass and each carbon. The locations of the extra centers were chosen arbitrarily, but keeping the molecular symmetry. Each one of these six centers has one $s$-type function with exponent 0.07 and one $p$-type function with exponent 0.22 . Figure 1 shows these seven extra centers labeled by $G$. The cartesian coordinates of the unique atoms used in the present calculations are shown in table I. This basis provided 10,032 CSFs for the $A^{\prime}$ symmetry and 9,019 CSFs for the $A^{\prime \prime}$ symmetry, with a total of 19,051 CSFs. The one particle basis set used in these calculations included 142 primitive Cartesian Gaussian functions contracted to 130 functions.

TABLE I: Cartesian coordinates of the unique atoms used in the present calculations (in units of $a_{0}$ ).

\begin{tabular}{lccc}
\hline \hline atom & $x$ & $y$ & $z$ \\
\hline $\mathrm{C}$ & 0.000000000 & 0.000000000 & 1.463563000 \\
$\mathrm{H}$ & 0.000000000 & -1.905867883 & 2.215033684 \\
$\mathrm{G}(\mathrm{CM})$ & 0.000000000 & 0.000000000 & 0.000000000 \\
$\mathrm{G}$ & 0.000000000 & -1.905867883 & 0.731781500 \\
\hline
\end{tabular}

The calculations with this larger basis was done in order to help to explain some discrepancies observed between our results and the available theoretical and experimental results, which seems to be due to the coupling of higher angular momentum caused by the use of $f$-type functions in the calculations of Sun et al. [16]. Since our codes deal only with $s$-, $p$-, and $d$-type functions, we need to include additional functions out of the molecular axis in order to obtain this type of coupling.

The computational cost of both calculations was almost the same. The calculations without extra centers used more CSFs than the calculations with extra centers, which means that the matrices to be inverted are bigger, the calculations being more extensive in terms of computational time and computational memory. On the other hand, the calculations with extra centers used more primitive functions and therefore the number of primitive integrals in these calculations was bigger than in the calculations without extra centers, being more time consuming.

\section{RESULTS AND DISCUSSION}

Figure 2 shows our calculated integral cross section in the static-exchange (SE) and in the static-exchange plus polarization (SEP) approximations. We also show in this figure the theoretical results of Sun et al. obtained with the complex Kohn method [16], also obtained in the SE and SEP approximations, and total experimental cross sections of Sueoka et al. [8], of Tanaka et al. [9] and of Szmytkowski and Krzysztofowicz [12]. In general there is good agreement between the two SE results. There is also good agreement between the two computed cross sections in the SEP approximation except for energies below $\sim 5 \mathrm{eV}$. For these energies the SEP results of Sun et al. present a shoulder and are greater than ours. As a consequence of this shoulder, the integral cross section of Sun et al. is closer to the experi- 


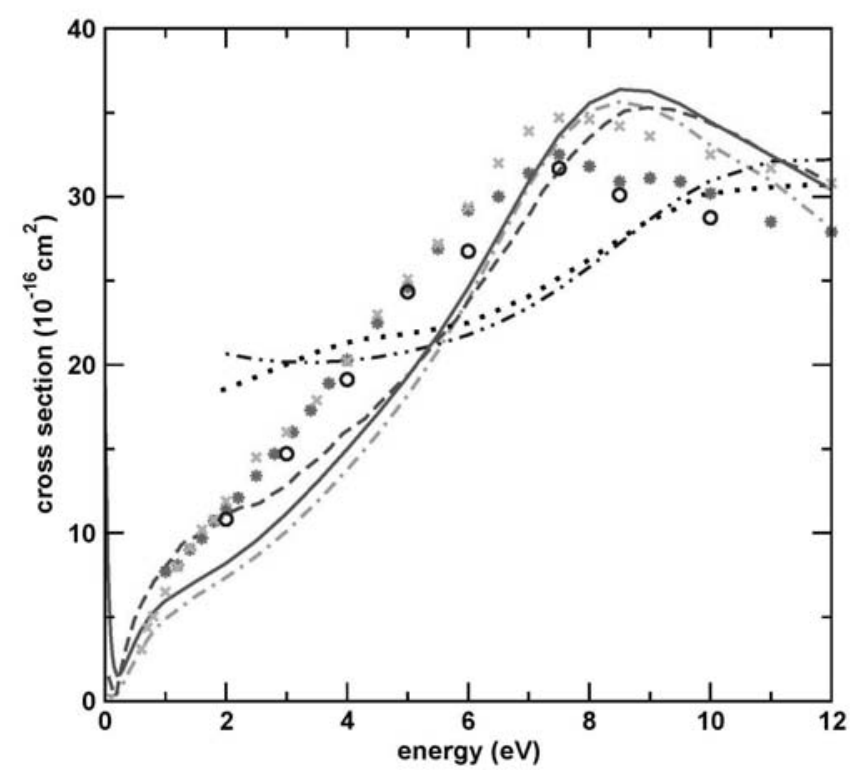

FIG. 2: Cross sections for ethane. Dotted (black) line, our results in the SE approximation; dot-dot-dashed line (blue), results of Ref. [16] in the SE approximation; dot-dashed line (green), our results in the SEP approximation; dashed line (red), results of Ref. [16] in the SEP approximation; stars (magenta), experimental cross section of Ref. [8]; circles (blue), experimental results of Ref. [9]; crosses (orange), results of Ref. [12]; solid (dark green) line, our results in the SEP approximation with the inclusion of extra centers (see text for discussion).

mental data than ours. We believe that the difference seen in the theoretical results occurs because we included only $s-$, $p$ - and $d$-type functions in the one-particle basis set while the complex Kohn results of Ref. [16] were obtained with a much larger basis set which included a substantial number of additional $s-, p-, d-$ and $f$-type functions on the molecular center of mass.

In order to investigate if the difference between the theoretical results is due to the one-particle basis, we performed another calculation including additional chargeless centers, as discussed above. This procedure would help in coupling higher angular momenta and therefore change the magnitude of the cross sections below $5 \mathrm{eV}$. This was done by the complex Kohn group by including the additional $s-, p-, d-$ and $f$-type functions on the molecular center of mass. The results obtained with these calculations are also shown in figure 2. The effect of improving the one-particle basis set in the ICS can be seen in figure 2. At low energy the ICS presents a rise that resembles the behavior of a virtual state. At around $0.2 \mathrm{eV}$ both calculations present a minimum. The minimum obtained with the inclusion of extra centers is shifted to higher energy and is larger when compared to the calculation with no extra centers. These two points will be discussed below.

Figures 3 and 4 show our calculated differential cross sections for energies from 2 to $10 \mathrm{eV}$ with and without the extra centers. We also show the results obtained by the complex Kohn group and also experimental results of Curry et al. [7],

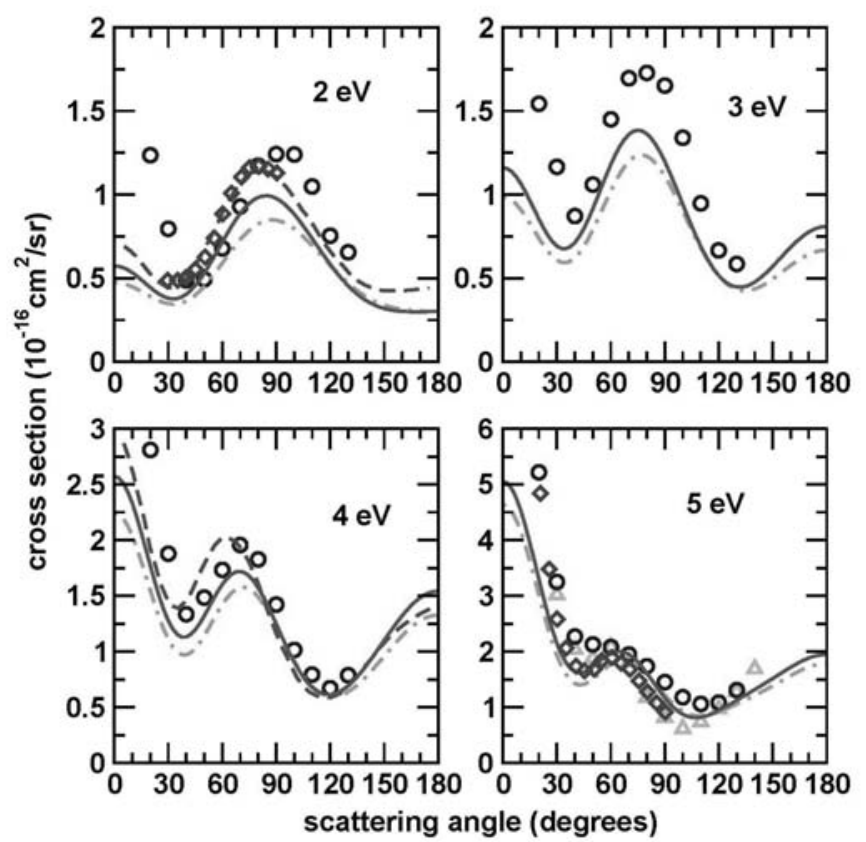

FIG. 3: Differential cross sections for ethane at 2, 3, 4 and $5 \mathrm{eV}$. Dot-dashed line (green), our computed SEP DCSs ; solid line (dark green), our computed SEP DCSs with extra centers; dashed line (red), theoretical results of Ref. [16]; circles (blue), experimental data of Ref. [9]; triangles (cyan), experimental data of Ref. [10] at $4.9 \mathrm{eV}$; diamonds (violet), results of Ref. [13] at 2 and $5.2 \mathrm{eV}$

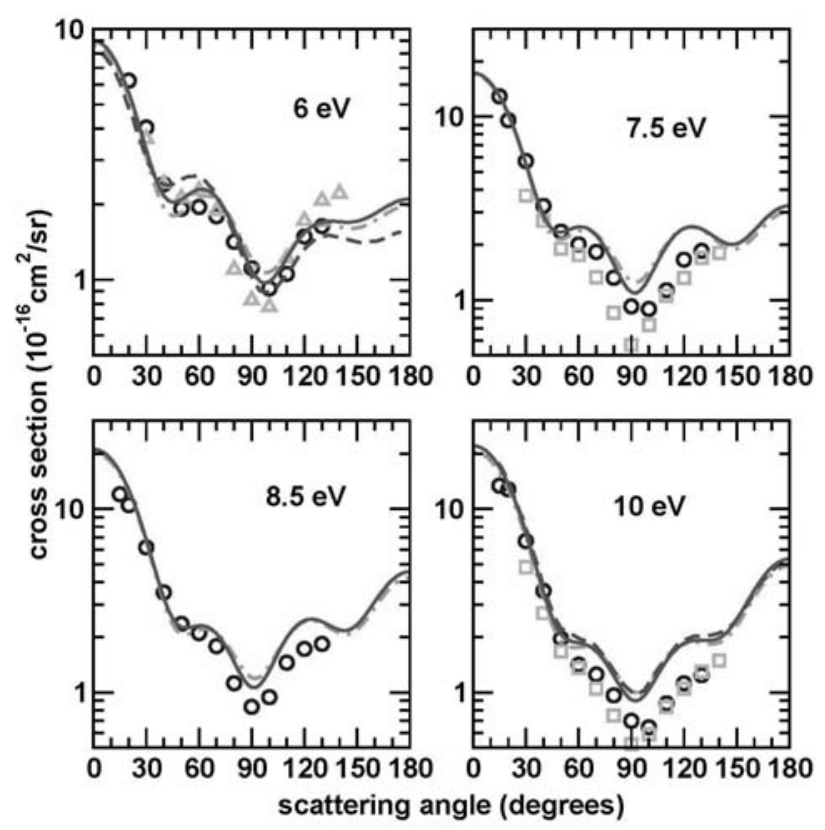

FIG. 4: As in Fig. 3 at 6, 7.5, 8.5 and $10 \mathrm{eV}$. Triangles (cian), results of Ref. [10] at 6 and $7.9 \mathrm{eV}$; squares (orange), experimental data of Ref. [7] 
Tanaka et al. [9], Mapstone et al. [10] and Merz and Linder [13]. For energies between 2 and $4 \mathrm{eV}$ our DCS without the extra centers lie below the complex Kohn results and the experimental data. Our results are improved by including the extra centers and the agreement with the complex Kohn results and with experiment becomes better. The results of the complex Kohn agree very well with the experimental data of Merz and Linder at $2 \mathrm{eV}$. For energies from 5 to $10 \mathrm{eV}$ our results agree well with the complex Kohn results and with the experimental data. At these energies the use of extra centers seems not to be as important as for lower energies. At 5 $\mathrm{eV}$ our results agree well with the experimental data of Merz and Linder for scattering angles above $\sim 30^{\circ}$.

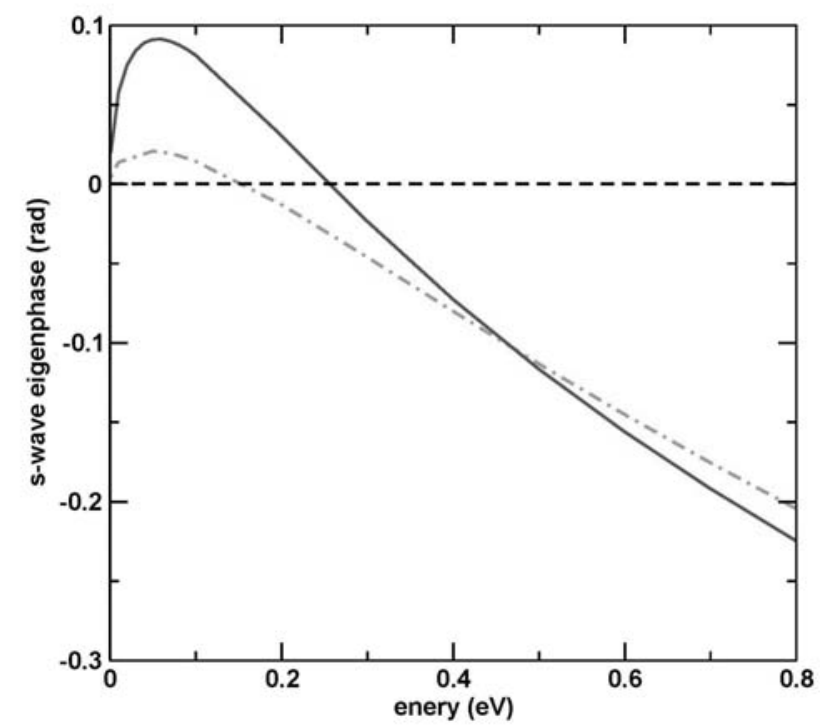

FIG. 5: $s$-wave eigenphase for $\mathrm{C}_{2} \mathrm{H}_{6}$. Dot-dashed (green) line, results obtained with no extra centers; solid (dark green) line, results obtained with extra centers.

Although the calculations including the extra centers have a little less CSFs than the calculations with no extra centers, the results obtained with the extra centers agree better with the experiment. This fact suggests that the small discrepancies seen between our results and the complex Kohn results and experimental data has origin in the one-particle basis set, which with the inclusion of extra functions couples higher angular momenta.

As discussed above our calculated integral cross sections show a minimum at around $0.2 \mathrm{eV}$. We computed the the $s$-wave eigenphase for both calculations, and the results are shown in figure 5. For the cross section obtained without the extra centers the $s$-wave eigenphase crosses zero below 0.2 $\mathrm{eV}$ while for the cross section obtained with the extra centers the $s$-wave eigenphase crosses zero above $0.2 \mathrm{eV}$. These results indicate a presence of a Ramsauer-Towsend minimum in the integral cross section of $\mathrm{C}_{2} \mathrm{H}_{6}$. The presence of this minimum has been reported by the complex Kohn calculations and by the experiments. Another point discussed above is that our results obtained with the extra centers show a rise at low energies which suggests a presence of a virtual state.

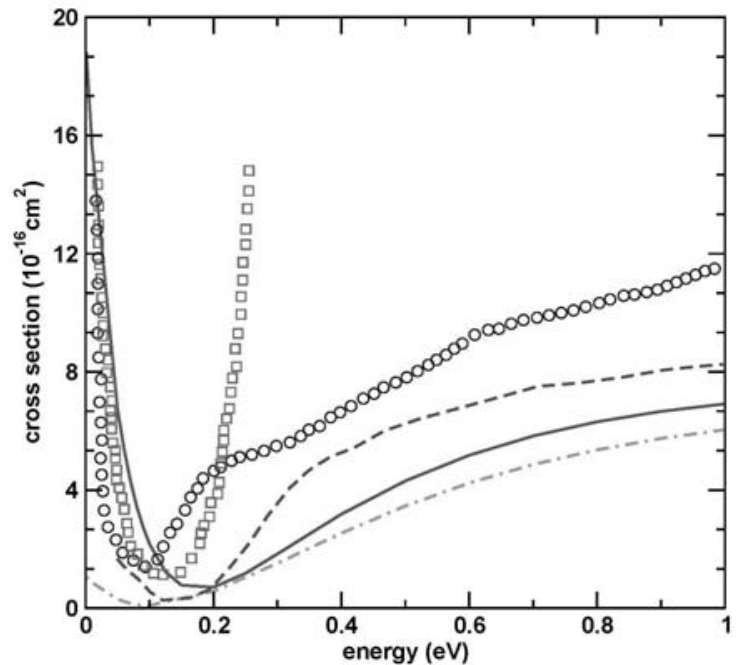

FIG. 6: Momentum transfer cross section for $\mathrm{C}_{2} \mathrm{H}_{6}$. As in figure 2, except circles (blue), results of Ref. [3]; squares (magenta), results of Ref. [5].

However, the behavior of the $s$-wave eigenphase shows that this rise is not related to a virtual state.

Figure 6 shows our calculated momentum transfer cross section with and without extra centers obtained in the SEP approximation compared to the complex Kohn results also obtained in the SEP approximation and to the experimental data of Duncan and Walker [3] and McCorkle et al. [5]. There is relative good agreement between our results and the other results shown in this figure.

\section{SUMMARY}

We presented elastic cross sections for electron collisions with ethane. Our results agree well with the experimental results available in the literature, although some differences between our results and the available theoretical and experimental results were seen for energies below $5 \mathrm{eV}$. Calculations using additional functions located on the center of mass and on centers out of the molecular axis provided results in better agreement with the available results for energies below $5 \mathrm{eV}$. Our results suggest that the small differences seen between our calculations, the complex Kohn calculations and the experimental data are due to the one-particle basis set, which is improved by including extra centers. Our computed integral cross section with the use of extra centers shows a rise at low energies. The behavior of the corresponding $s$ wave eigenphase indicates that this rise is not related to a virtual state. In particular, our results show a broad maximum around $8.5 \mathrm{eV}$ and the existence of a Ramsauer-Townsend minimum around $0.2 \mathrm{eV}$, in agreement with the experimental predictions and with theoretical results. 


\section{Acknowledgments}

M.H.F.B. and M.A.P.L. acknowledge support from Conselho Nacional de Desenvolvimento Científico e Tecnológico (CNPq). R.F. da C. and M.A.P.L. would like to acknowledge the financial support from the Brazilian agency Fundação de Amparo à Pesquisa do Estado de São Paulo
(FAPESP). M.H.F.B. acknowledges support from the Paraná State agency Fundação Araucária and from Finep. The authors acknowledge computational support from CENAPADSP. M.H.F.B. acknowledges computational support from Prof. Carlos de Carvalho at DFis-UFPR. Discussions with Dr. Alexandra P.P. Natalense are greatfully acknowledged.
[1] B. Boudaïffa, P. Cloutier, D. Hunting, M. A. Huels, and L. Sanche, Science 287, 1658 (2000)

[2] See, for example, C. Winstead and V. McKoy, J. Chem. Phys. 125, 074302 (2006); D. Bouchiha, J. D. Gorfinkiel, L. G. Caron, and L. Sanche, J. Phys. B: At. Mol. Opt. Phys. 39, 975 (2006); T. N. Rescigno, C. S. Trevisan, and A. E. Orel, Phys. Rev. Lett. 96, 213201 (2006); M. Allan, J. Phys. B 39, 2939 (2006); M. H. F. Bettega and M. A. P. Lima, J. Chem. Phys. 126, 194317 (2007), and references therein.

[3] C. W. Duncan and I. C. Walker, J. Chem. Soc. Faraday II 70, 577 (1974).

[4] L. G. Christophorou and D. L. McCorkle, Can. J. Chem. 55, 1876 (1977).

[5] D. L. McCorkle, L. G. Christophorou, D. V. Maxey, and J. G. Carter, J. Phys. B 11, 3067 (1978).

[6] K. Floeder, D. Fromme, W. Raith, A. Schwab and G. Sinapius, J. Phys. B 18, 3347 (1985).

[7] P. J. Curry, W. R. Newell, and A. C. H. Smith, J. Phys. B 18, $2303(1985)$

[8] 0. Sueoka and S. Mori, J. Phys. B 19, 4035 (1986).

[9] H. Tanaka, L. Boesten, D. Matsunaga, and T. Kudo, J. Phys. B 21, 1255 (1988)

[10] B. Mapstone and W. R. Newell, J. Phys. B 25, 491 (1992).

[11] H. Nishimura and H. Tawara, J. Phys. B 24, L363 (1991).

[12] C. Szmytkowski and A. M. Krzysztofowicz, J. Phys. B 28, 4291 (1995).

[13] R. Merz and F. Linder, J. Phys. B 31, 4663 (1998).

[14] S. L. Lunt, J. Randell, J.-P. Ziesel, G. Mrotzek, and D. Field, J. Phys. B 31, 4225 (1998).
[15] C. Winstead, P. G. Hipes, M. A. P. Lima, and V. McCoy, J. Chem. Phys. 94, 5455 (1991).

[16] W. Sun, C. W. McCurdy, and B. H. Lengsfield III, J. Chem. Phys. 97, 5480 (1992).

[17] M. H. F. Bettega, A. J. S. Oliveira, A. P. P. Natalense, M. A. P. Lima, and L. G. Ferreira, Eur. Phys. J. D 1, 291 (1998).

[18] K. Takatsuka and V. McKoy, Phys. Rev. A 24, 2473 (1981); Phys. Rev. A 30, 1734 (1984).

[19] M. H. F. Bettega, L. G. Ferreira, and M. A. P. Lima, Phys. Rev. A 47, 1111 (1993).

[20] CRC Handbook of Chemistry and Physics, 79th ed., edited by D. R. Lide (CRC, Boca Raton, 1998).

[21] B. M. Bode and M. S. Gordon, J. Mol. Graphics Mod. 16, 133 (1998).

[22] G. B. Bachelet, D. R. Hamann, and M. Schlüter, Phys. Rev. B 26, 4199 (1982).

[23] M. H. F. Bettega, A. P. P. Natalense, M. A. P. Lima, and L. G Ferreira, Int. J. Quantum Chem. 60, 821 (1996).

[24] T. H. Dunning Jr., J. Chem. Phys. 53, 2823 (1970)

[25] C. Winstead and V. McKoy, Phys. Rev. A 57, 3589 (1998); C. Winstead, V. McKoy, and M. H. F. Bettega Phys. Rev. A 72, 042721 (2005)

[26] C. W. Bauschlicher Jr., J. Chem. Phys. 72, 880 (1980).

[27] M. H. F. Bettega, R. F. da Costa, and M. A. P. Lima, Phys. Rev. A 77, 052706 (2008).

[28] A. R. Lopes, M. H. F. Bettega, M. A. P. Lima, and L. G. Ferreira, J. Phys. B 37, 997 (2004). 